\title{
Phase diagram of the fully frustrated transverse-field Ising model on the honeycomb lattice
}

\author{
T. Coletta, ${ }^{1}$ J.-D. Picon, ${ }^{1}$ S. E. Korshunov, ${ }^{2}$ and F. Mila ${ }^{1}$ \\ ${ }^{1}$ Institute of Theoretical Physics, École Polytechnique Fédérale de Lausanne, CH-1015 Lausanne, Switzerland \\ ${ }^{2}$ L. D. Landau Institute for Theoretical Physics, 142432 Chernogolovka, Russia
}

(Received 28 June 2010; published 3 February 2011)

\begin{abstract}
Motivated by the current interest in the quantum dimer model on the triangular lattice, we investigate the phase diagram of the closely related fully frustrated transverse-field Ising model on the honeycomb lattice using classical and semiclassical approximations. We show that, in addition to the fully polarized phase at a large field, the classical model possesses a multitude of phases that break the translational symmetry which, in the dimer language, correspond to a plaquette phase and a columnar phase separated by an infinite cascade of mixed phases. The modification of the phase diagram by quantum fluctuations has been investigated in the context of linear spin-wave theory. The extrapolation of the semiclassical energies suggests that the plaquette phase extends down to zero field for spin $1 / 2$, in agreement with the $\sqrt{12} \times \sqrt{12}$ phase of the quantum dimer model on the triangular lattice with only kinetic energy.
\end{abstract}

DOI: 10.1103/PhysRevB.83.054402

PACS number(s): 05.50.+q, 71.10.-w, 75.10.Jm

\section{INTRODUCTION}

Quantum dimer models have emerged as one of the main paradigms in the investigation of quantum spin liquids. The Rokhsar-Kivelson (RK) quantum dimer model (QDM), which includes a potential interaction of amplitude $V$ between dimers facing each other and a kinetic term of amplitude $t$ flipping them around rhombic plaquettes, has recently attracted special attention. The main reason comes from the presence on the triangular lattice of a resonating valence bond (RVB) phase first discovered by Moessner and Sondhi ${ }^{1}$ and extensively studied since then using zero-temperature Green's function quantum Monte Carlo (GFQMC). ${ }^{2-4}$ Exact results have been obtained at the RK point $(V / t=1)$, where the sum of all configurations can be proven to be a ground state, ${ }^{1}$ and at $V>t$, where the nonflippable configurations are the ground states. Analytical results have also been obtained in the limit $V / t \rightarrow-\infty$, where columnar states have been shown to be selected. However, in the intermediate range below the RK point, most of what is known about the model is based on numerical simulations.

A closely related model for which a number of analytical results have already been obtained is the fully frustrated transverse-field Ising model (FFTFIM) on the honeycomb lattice defined by the Hamiltonian:

$$
H=-\frac{J}{S^{2}} \sum_{\langle i, j\rangle} M_{i j} S_{i}^{z} S_{j}^{z}-\frac{\Gamma}{S} \sum_{i} S_{i}^{x},
$$

where $\Gamma>0$ is the transverse magnetic field, $J>0$ is the coupling constant of the Ising interaction term, $\langle i, j\rangle$ denotes pairs of nearest neighbors on the honeycomb lattice, and $M_{i j}= \pm 1$ is such that for each hexagon of the lattice the number of antiferromagnetic bonds $\left(M_{i j}=-1\right)$ is odd, with different choices of $M_{i j}$ corresponding to the same model up to the rotation of some spins by $\pi$ around the $x$ axis. ${ }^{5}$ Transverse-field Ising models have been the subject of intense investigations over the years. ${ }^{6}$ The relationship between the FFTFIM on a regular lattice and the QDM on the dual lattice was first emphasized by Moessner et al., ${ }^{7}$ who showed (see also Ref. 1) that, in the limit $\Gamma / J \rightarrow 0$, the FFTFIM on the honeycomb lattice maps to the QDM on the triangular lattice with $t=\Gamma^{2} / J$ and $V=0$. For the FFTFIM on the honeycomb lattice, they also carried out a Landau-Ginzburg analysis and identified four soft modes that simultaneously become gapless when $\Gamma / J$ decreases, leading to a surprisingly large unit cell of 48 sites. Details of this calculation have been reported by Moessner and Sondhi. ${ }^{8}$ These authors further conjectured that the translational symmetry-breaking transition out of the paramagnetic phase coming from large $\Gamma / J$ provides a reasonable description of the transition between the RVB phase and the intermediate phase of the QDM on the triangular lattice. $^{1}$

Building on this conjecture, Misguich and one of the present authors have carried out a semiclassical investigation of the paramagnetic phase of the FFTFIM on the honeycomb lattice ${ }^{9}$ and have shown that the dispersion of the spin waves and their softening at the transition are in remarkable agreement with the dispersion of visons in the QDM on the triangular lattice and their crystallization transition as revealed by quantum Monte Carlo (QMC) simulations. ${ }^{4}$ However, the analysis of Ref. 9 has not covered the small $\Gamma / J$ parameter range.

In the present paper, we perform a systematic investigation of the FFTFIM on the honeycomb lattice in the complete parameter range $0 \leqslant \Gamma / J<+\infty$ with classical and semiclassical approximations. As we shall see, the classical phase diagram is much richer than expected, with an infinite number of different crystalline phases below the paramagnetic phase: a plaquette phase, a cascade of mixed phases, and a highly degenerate columnar phase. Quantum fluctuations have been treated within linear spin-wave theory, leading to a partial lifting of the degeneracy of the columnar phase and to an increase in the size of the region occupied by the plaquette phase.

To make contact between the physics of the FFTFIM and of the QDM, it is useful to introduce a gauge theory defined on the triangular lattice by the Hamiltonian:

$$
H=-J \sum_{l} \tau_{l}^{x}-\Gamma \sum_{i} \prod_{l(i)} \tau_{l(i)}^{z},
$$

where $i$ runs over the sites of the dual honeycomb lattice and $l(i)$ are the three bonds forming the triangular plaquette around site $i$. As shown by Moessner et al., ${ }^{10}$ the FFTFIM is 
equivalent, up to a twofold degeneracy, to the odd sector of this gauge theory defined by

$$
\prod_{l[a]} \tau_{l[a]}^{x}=-1,
$$

for all $a$, where $a$ is a site of the triangular lattice and the product over $l[a]$ runs over the six links emanating from $a$. For a succinct discussion of the correspondence between the three models, see, e.g., the introduction of Ref. 9.

The discussion of the ordered phases is simpler in the context of the gauge theory. Indeed, in the FFTFIM language, the actual orientation of the spins in a given state depends on the choice of the matrix $M_{i j}$. In contrast, the dimer operator of the gauge theory defined by

$$
d_{l}=\frac{1}{2}\left(1-\tau_{l}^{x}\right)
$$

translates into

$$
d_{i j}=\frac{1}{2}\left(1-M_{i j} \frac{S_{i}^{z} S_{j}^{z}}{S^{2}}\right)
$$

in the Ising language, and its expectation value does not depend on the choice of $M_{i j}$. Another advantage of the gauge-invariant language is that it allows us to make a direct comparison with the numerical results obtained on the QDM since they live on the same lattice and are defined in terms of the same link operators. So, while all reasonings and calculations will be performed in the context of the FFTFIM, the only formulation adapted to the semiclassical approach, the structures of different ordered phases will also be described in gauge-invariant terms. Throughout this paper, we use only gauges in which each hexagon of the lattice contains exactly one antiferromagnetic bond (with $M_{i j}=-1$ ) and five ferromagnetic bonds (with $M_{i j}=+1$ ). Most results will be presented for the simplest periodic arrangement of the antiferromagnetic bonds shown in Fig. 1. However, this choice of gauge does not always lead to the smallest possible unit cell in terms of the spin representation. Thus, we will also introduce other gauges whenever this is helpful.

This paper is organized as follows. In Sec. II, we concentrate on the limit $\Gamma / J \ll 1$, which has not been considered in Refs. 1,7-9, and we show that columnar phases reminiscent of the $V \rightarrow-\infty$ limit of the QDM are stabilized. In Sec. III, we revisit the vicinity of the RVB phase. We recover the symmetry predicted by the Landau-Ginzburg approach of Refs. 7 and 8 and by the spin-wave analysis of Ref. 9, but we find that the bonds with the largest dimer density form separate four-site

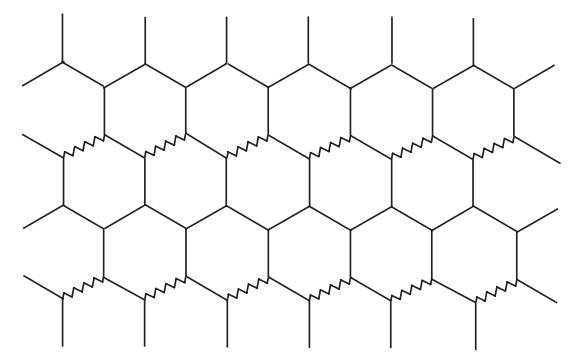

FIG. 1. Sketch of the gauge used in most of this paper. Here and in other figures antiferromagnetic bonds (with $M_{i j}=-1$ ) are shown by zigzags, with all other bonds being ferromagnetic (with $M_{i j}=+1$ ). rhombic plaquettes instead of having a uniform distribution inside a 12-site unit cell as reported in Ref. 9. The reasons for this discrepancy are explained in Sec. IIIC. In Sec. IV, we discuss the transition between the plaquette phase and the columnar phase and show that they are separated by a region of intermediate phases of mixed character. The stability of these phases with respect to quantum fluctuations and the semiclassical phase diagram are discussed in Sec. V. A short conclusion is given in Sec. VI.

\section{COLUMNAR PHASE}

In this section we discuss the properties of the model when $\Gamma / J$ is small. The argument proceeds in three steps. First, we determine the ground-state manifold of the Heisenberg model with purely Ising-like interactions in the absence of magnetic field $(\Gamma=0)$. Then, we investigate how the extensive degeneracy of these ground states is lifted by a small transverse field. Finally, we discuss the effect of quantum fluctuations in the context of linear spin-wave theory.

\section{A. Zero transverse field}

In the absence of a transverse magnetic field $(\Gamma=0)$, we are left with a model without quantum fluctuations in which the interaction term couples only the $z$ components of neighboring spins on the honeycomb lattice. With our choice of gauge, one bond on each hexagon is antiferromagnetic $\left(M_{i j}=-1\right)$, and the others are ferromagnetic $\left(M_{i j}=1\right)$. Frustration is present since it is clearly impossible to minimize the energy of all bonds of a given hexagon.

For Ising spins, i.e., spins that can only point up or down along the $z$ direction, the best one can do is to satisfy five bonds, leaving one bond unsatisfied. This can be done in six different ways according to which bond is not satisfied ("frustrated"), and the resulting energy is $-4 J$. Up to a global reversal of the spins, a ground state is characterized by the distribution of frustrated bonds such that there is exactly one of them per hexagon.

For three-dimensional vectors of norm $S$, the situation is slightly more subtle because the 12 Ising configurations with all spins parallel or antiparallel to the $z$ axis are not the only ground states of a single hexagon. To see this, let us consider a single hexagon and investigate the possibility of a given spin $i$ not to be directed along $z$. The variation of the energy of the hexagon

$$
E_{\mathrm{hex}}=-J \sum_{j=1}^{6} M_{j, j+1} \cos \theta_{j} \cos \theta_{j+1}
$$

(where the angle $\theta_{j}$ parameterizes the deviation of spin $j$ from the $z$ axis) with respect to $\theta_{i}$ leads to the condition

$$
M_{i-1, i} \cos \theta_{i-1}+M_{i, i+1} \cos \theta_{i+1}=0 .
$$

If this condition is satisfied, the terms in Eq. (6) that depend on $\theta_{i}$ drop out, so that one is left with the energy of an open chain of five spins. In an open chain one can trivially minimize the energy of each bond by choosing $\cos \theta_{j+1}=M_{j, j+1} \cos \theta_{j}=$ \pm 1 , which leads to $E=-4 J$ and to the automatic fulfillment of condition (7), leaving $\theta_{i}$ arbitrary. Note that this argument excludes a deviation from the $z$ axis of more than one spin 
since the energy of a five-spin open chain cannot be as low as $-4 J$ if not all five spins are along $z$. So, for three-dimensional spins, the energy of a single hexagon is minimal as soon as it is minimal for four consecutive bonds, and the spin at the remaining site can have any direction.

It is natural to ask whether this additional freedom increases the degeneracy of the ground-state manifold of the continuous model in comparison with the case of Ising spins. To demonstrate that this is not the case, let us assume that at site $i$ the spin is not along $z$. To minimize simultaneously the energy of the three hexagons to which it belongs, three conditions of the form (7) must be fulfilled:

$$
\begin{aligned}
& Y_{1}+Y_{2}=0, \\
& Y_{2}+Y_{3}=0, \\
& Y_{3}+Y_{1}=0
\end{aligned}
$$

where $Y_{a}=M_{i, i_{a}} \cos \theta_{i_{a}}= \pm 1$ (with $a=1,2,3$ ) and $i_{a}$ are the three nearest neighbors of site $i$. It is evident that the restriction $Y_{a}= \pm 1$ does not allow all three Eqs. (8) to be satisfied simultaneously. Therefore, it is impossible for any spin not to point along $z$, and the ground-state manifold coincides with that of the frustrated Ising model with the same lattice, i.e., it consists of all Ising configurations with one frustrated bond per hexagon. Each of these states is a local minimum of the Hamiltonian.

\section{B. Classical ground states in a small transverse field}

Let us now switch on a small transverse field and study how the local minima of the classical Hamiltonian evolve as a function of the field. Since the field is along $x$, the spins are expected to acquire a small $x$ component, and to describe the spin configuration evolving from a given ground state of the pure Ising case, we use the parametrization

$$
\begin{gathered}
S_{i}^{x}=S \sin \theta_{i}, \\
S_{i}^{z}=\sigma_{i} S \cos \theta_{i},
\end{gathered}
$$

where $\sigma_{i}= \pm 1$ is the sign of $S_{i}^{z}$ and is determined by the ground state of the pure Ising case around which we expand. In terms of the gauge-invariant bond variable $\tau_{i j}=M_{i j} \sigma_{i} \sigma_{j}$, which is equal to $-1(+1)$ if the bond $\langle i, j\rangle$ is frustrated (not frustrated), the classical energy can be rewritten as

$$
E=-J \sum_{\langle i, j\rangle} \tau_{i j} \cos \theta_{i} \cos \theta_{j}-\Gamma \sum_{i} \sin \theta_{i} .
$$

In the limit $\Gamma \ll J$ the deviations from the $z$ direction are small, and the classical energy can be expanded in the variables $\theta_{i}$ around $\theta_{i}=0$. To second order, the interaction term in Eq. (10) decouples: $\tau_{i j} \cos \theta_{i} \cos \theta_{j} \approx \tau_{i j}\left(1-\theta_{i}^{2} / 2-\theta_{j}^{2} / 2\right)$. Now, for any ground state of the pure Ising case, the set $\left\{\tau_{i j}\right\}$ is such that only one bond in each hexagon is frustrated. Therefore, each site belongs at most to one frustrated bond. If we denote by $\mathrm{F}$ (NF) the set of what we call frustrated (nonfrustrated) sites, namely, the sites belonging to one frustrated bond (no frustrated bond), the energy up to second order can be rewritten:

$$
E^{(2)}=E_{\Gamma=0}+\sum_{i \in \mathrm{F}}\left(\frac{J}{2} \theta_{i}^{2}-\Gamma \theta_{i}\right)+\sum_{i \in \mathrm{NF}}\left(\frac{3 J}{2} \theta_{i}^{2}-\Gamma \theta_{i}\right) .
$$

Minimizing $E^{(2)}$ with respect to $\left\{\theta_{i}\right\}$ leads to

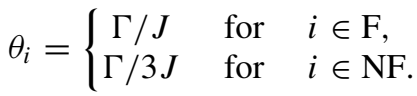

Since the number of frustrated and nonfrustrated sites is the same for all ground states, the energy up to second order in $\theta_{i}$ is the same in all ground states. So second-order corrections do not lift the degeneracy. They only induce a difference in orientation between the spins that belong to a frustrated bond and those that do not.

So to lift the degeneracy, we have to push the expansion in $\theta_{i}$ to higher orders. To fourth order, it reads

$$
\begin{aligned}
E^{(4)}= & E_{\Gamma=0}+\sum_{i \in \mathrm{F}}\left[J\left(\frac{\theta_{i}^{2}}{2}-\frac{\theta_{i}^{4}}{4 !}\right)-\Gamma\left(\theta_{i}-\frac{\theta_{i}^{3}}{3 !}\right)\right] \\
& +\sum_{i \in \mathrm{NF}}\left[3 J\left(\frac{\theta_{i}^{2}}{2}-\frac{\theta_{i}^{4}}{4 !}\right)-\Gamma\left(\theta_{i}-\frac{\theta_{i}^{3}}{3 !}\right)\right] \\
& -\frac{J}{4} \sum_{\langle i, j\rangle} \tau_{i j} \theta_{i}^{2} \theta_{j}^{2} .
\end{aligned}
$$

From the previous discussion, we know that the values of $\theta_{i}$ minimizing the energy to order $O\left(\theta^{2}\right)$ are given by Eq. (12). Injecting these solutions into the fourth-order expansion of the energy, we notice that the terms $\theta_{i}^{3}$ and $\theta_{i}^{4}$ only contribute in two different ways, depending on the type of site (frustrated or nonfrustrated). They will thus not lift the degeneracy. In contrast, the cross terms $\tau_{i j} \theta_{i}^{2} \theta_{j}^{2}$ contribute in four different ways, depending on the environment of sites $i$ and $j$. The four cases are illustrated in Fig. 2.

The contributions of the fourth-order cross terms to the energy for the different configurations in units of $\Gamma^{4} / 4 J^{3}$ are +1 for Fig. 2(a), $-\frac{1}{81}$ for Fig. 2(b), $-\frac{1}{9}$ for Fig. 2(c),

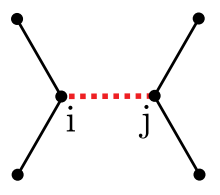

(a)

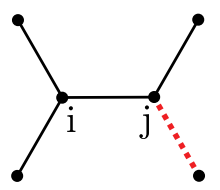

(c)

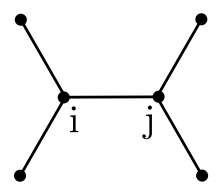

(b)

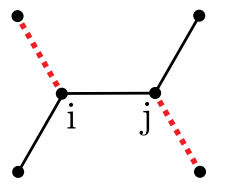

(d)
FIG. 2. (Color online) Local configurations of frustrated bonds leading to different contributions of the fourth-order cross term $-\frac{J}{4} \tau_{i j} \theta_{i}^{2} \theta_{j}^{2}$. (a) The same frustrated bond contains sites $i$ and $j$; (b) No frustrated bond contains $i$ or $j$; (c) One frustrated bond contains either $i$ or $j$; (d) One frustrated bond contains $i$, and another one contains $j$. 


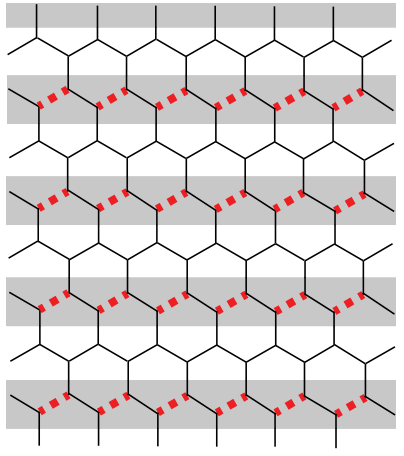

(a) first columnar state

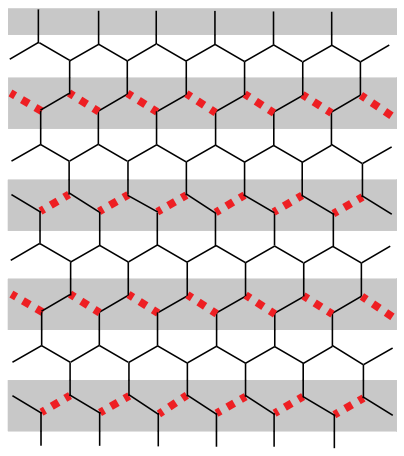

(b) second columnar state

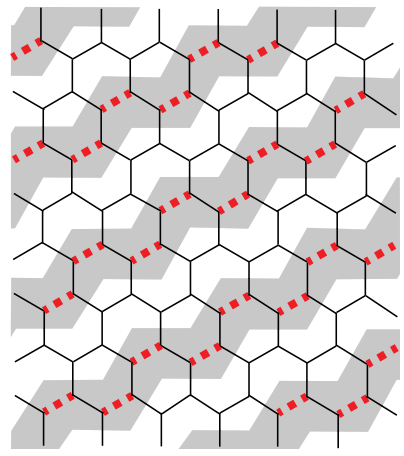

(c) third columnar state

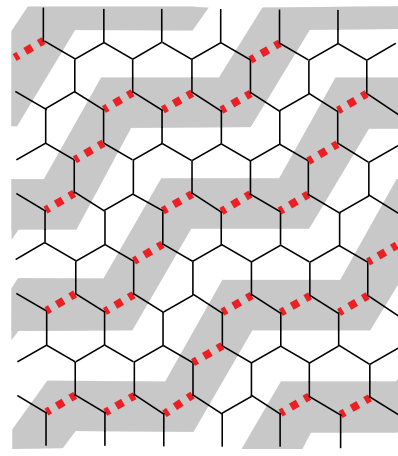

(d) fourth columnar state

FIG. 3. (Color online) Examples of columnar states. (a) Columnar state without domain walls; (b) Columnar state with the highest possible density of horizontal domain walls; (c) Columnar state with the highest possible density of domain walls perpendicular to the frustrated bonds; (d) Columnar state that differs from the third one by having two times less domain walls. The frustrated bonds are represented as dashed red lines. In the dimer representation, the bonds of the dual triangular lattice that intersect the frustrated bonds of the honeycomb lattice have the highest dimer density.

and -1 for Fig. 2(d). Since these energies are not equal, these cross terms are expected to lift the degeneracy, at least partially.

For a lattice of $N_{\text {hex }}$ hexagons the total number of bonds is $3 N_{\text {hex }}$. The constraint that each hexagon has one frustrated bond implies that the number of frustrated bonds is equal to $N_{\text {hex }} / 2$. This fixes the number $N_{a}$ of configurations in Fig. 2(a) to be equal to $N_{\text {hex }} / 2$. In contrast, the numbers of configurations of the types depicted in Figs. 2(b), 2(c), and 2(d) $\left(N_{b}, N_{c}\right.$, and $N_{d}$, respectively) depend on the way the frustrated bonds are arranged on the lattice. However, $N_{b}, N_{c}$, and $N_{d}$ are not independent but have to satisfy the following relations:

$$
\begin{gathered}
N_{b}+N_{c}+N_{d}=\frac{5}{2} N_{\mathrm{hex}}, \\
N_{a}=\frac{1}{4}\left(N_{c}+2 N_{d}\right) .
\end{gathered}
$$

Equation (14) comes from the conservation of the total number of bonds $N_{a}+N_{b}+N_{c}+N_{d}=3 N_{\text {hex }}$, whereas the right-hand side of Eq. (15) comes from counting all frustrated bonds by looking at how many of them are adjacent to each of the nonfrustrated bonds. The result of this calculation has to be divided by 4 because in such a procedure each frustrated bond is counted four times.

The total contribution of the fourth-order cross terms of the energy can then be written as

$$
\begin{aligned}
-\frac{J}{4} \sum_{\langle i, j\rangle} \tau_{i j} \theta_{i}^{2} \theta_{j}^{2} & \approx \frac{J}{4}\left(\frac{\Gamma}{J}\right)^{4}\left(N_{a}-N_{d}-\frac{1}{9} N_{c}-\frac{1}{81} N_{b}\right) \\
& \approx \frac{J}{4}\left(\frac{\Gamma}{J}\right)^{4}\left(\frac{22}{81} N_{\mathrm{hex}}-\frac{64}{81} N_{d}\right) .
\end{aligned}
$$

This contribution is a decreasing function of $N_{d}$, so the lowest energy will be reached for the largest possible value of $N_{d}$. Now, since there is only one frustrated bond per hexagon, $N_{d}$ cannot exceed the number of frustrated bonds, $N_{a}=N_{\text {hex }} / 2$. This upper limit is reached for configurations in which all the frustrated bonds are organized into chains of alternating frustrated and nonfrustrated bonds (see the examples in Fig. 3).
In what follows we refer to this family of states as columnar states (see Fig. 3). In columnar states, Eqs. (14) and (15) fix both $N_{b}$ and $N_{c}$ to be equal to $N_{\text {hex }}$.

So the fourth-order contribution to the energy partially lifts the degeneracy and selects the family of columnar states. A priori, higher orders might further lift the degeneracy. That this is not the case is best seen by constructing the exact local minima that correspond to columnar states. We start by rewriting the energy:

$$
\begin{aligned}
E= & -\sum_{i \in \mathrm{NF}}\left[\frac{J}{2} \cos \theta_{i}\left(\cos \theta_{i_{1}}+\cos \theta_{i_{2}}+\cos \theta_{i_{3}}\right)+\Gamma \sin \theta_{i}\right] \\
& -\sum_{j \in \mathrm{F}}\left[\frac{J}{2} \cos \theta_{j}\left(-\cos \theta_{j_{1}}+\cos \theta_{j_{2}}+\cos \theta_{j_{3}}\right)+\Gamma \sin \theta_{j}\right],
\end{aligned}
$$

where $i_{1}, i_{2}, i_{3}\left(j_{1}, j_{2}, j_{3}\right)$ are the three neighbors of site $i(j)$ and the frustrated bond is taken to be between sites $j$ and $j_{1}$. To minimize the energy, the set of angles $\left\{\theta_{i}, \theta_{j}\right\}$ must be a solution of the following equations:

$$
\begin{aligned}
& \frac{\partial E}{\partial \theta_{i}}=J \sin \theta_{i}\left(\cos \theta_{i_{1}}+\cos \theta_{i_{2}}+\cos \theta_{i_{3}}\right)-\Gamma \cos \theta_{i}=0, \\
& \frac{\partial E}{\partial \theta_{j}}=J \sin \theta_{j}\left(-\cos \theta_{j_{1}}+\cos \theta_{j_{2}}+\cos \theta_{j_{3}}\right)-\Gamma \cos \theta_{j}=0 .
\end{aligned}
$$

Now, in columnar structures, all frustrated sites have identical environments (with exactly two frustrated neighbors), and all unfrustrated sites also have identical environments (with exactly one frustrated neighbor). So, if angles $\theta_{1}$ and $\theta_{2}$ satisfy the equations

$$
\begin{gathered}
J \sin \theta_{1}\left(2 \cos \theta_{1}+\cos \theta_{2}\right)-\Gamma \cos \theta_{1}=0, \\
J \sin \theta_{2} \cos \theta_{1}-\Gamma \cos \theta_{2}=0,
\end{gathered}
$$

then the set of angles

$$
\theta_{i}=\left\{\begin{array}{lll}
\theta_{1} & \text { for } & i \in \mathrm{NF} \\
\theta_{2} & \text { for } & i \in \mathrm{F}
\end{array}\right.
$$


is a solution of Eqs. (18). The nontrivial solutions of Eqs. (19) describing the evolution of columnar states with the change of $\Gamma / J$ are given by

$$
\sin \theta_{1}=\frac{\sin (\beta / 3)}{\cos (\beta)}, \quad \sin \theta_{2}=\frac{\sin (\beta)}{\cos (\beta / 3)},
$$

where $\tan \beta=\Gamma / J$.

Substituting Eq. (20) into Eq. (10) shows that the classical energy of a columnar state is given by

$$
E_{\mathrm{col}}=-\frac{N}{2}\left[J \cos \theta_{1}\left(\cos \theta_{1}+\cos \theta_{2}\right)+\Gamma\left(\sin \theta_{1}+\sin \theta_{2}\right)\right],
$$

where $N$ is the total number of sites. Naturally, the variation of $E_{\text {col }}$ with respect to $\theta_{1}$ and $\theta_{2}$ reproduces Eqs. (19), which we used to find the values of $\theta_{1}$ and $\theta_{2}$. In order to verify that it never becomes more advantageous to minimize $N_{d}$ rather then to maximize it, we have also studied the solutions with $N_{d}=0$ and checked that for any relation between $\Gamma$ and $J$ they have higher energy than the columnar states (see the Appendix).

A convenient classification of columnar states can be introduced by describing them in terms of zero-energy domain walls formed on the background of the simplest columnar state, an example of which is shown in Fig. 3(a). We call it the first columnar state. In this state all frustrated bonds have the same orientation and form straight columns, as shown in Fig. 3 by the shading. In terms of Fig. 3 the walls of the first type are horizontal and take place whenever the orientation of the frustrated bonds changes from left to right. The second columnar state [Fig. 3(b)] corresponds to the configuration having the highest possible density of such domain walls.

The domain walls of the second type are perpendicular to the frustrated bonds, and correspond to changing the orientation not of frustrated bonds but of columns. The third columnar state [Fig. 3(c)] is the configuration having the highest possible density of walls of the second type as the orientation of the columns changes at every frustrated bond. Other columnar states having the same classical energy can be obtained by introducing arbitrary sequences of parallel domain walls either of the first or of the second type separating domains of the first columnar state. An analogous classification of columnar states was introduced by Moessner and Sondhi ${ }^{8}$ in terms of the QDM.

Figure 4(a) presents a plot of the dimer density for the first columnar state at $\Gamma / J=1.5$. The bonds of the dual triangular lattice having the highest dimer densities are organized into a columnar pattern. Figure 4(b) shows the first columnar state in the classical spin model. The $S^{z}$ component of the spin on frustrated sites [green (dark gray) arrows in Fig. 4(b)] is smaller than that on nonfrustrated sites.

\section{Quantum fluctuations}

The effect of quantum fluctuations on the columnar states, in particular their local stability and their degeneracy, has been investigated in the context of linear spin-wave theory (LSWT). It is impossible to perform a LSWT calculation for all columnar states since the family is infinite and contains many members that are not periodic. The logic we have followed is

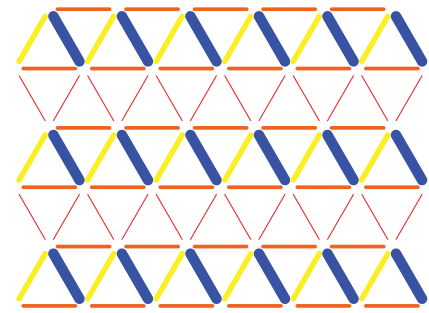

(a) $1^{\text {st }}$ Columnar state: dimer representation

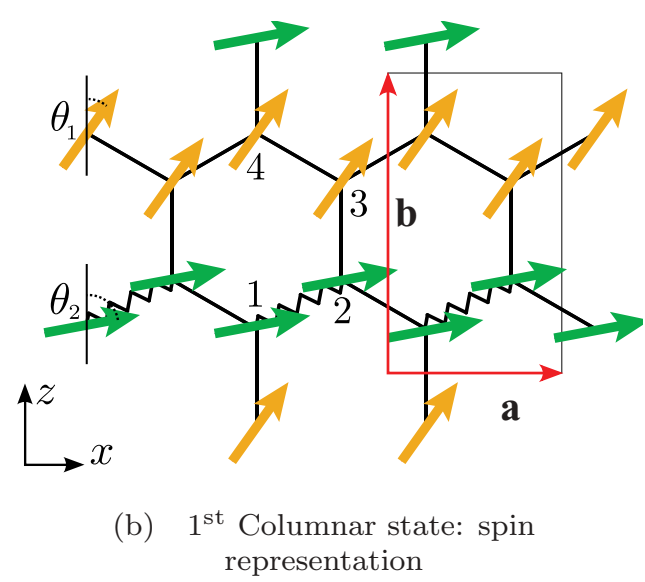

FIG. 4. (Color online) (a) Plot of the dimer density $d_{i j}$ at $\Gamma / J=1.5$ for the first columnar state. The thickness of the bonds is proportional to $d_{i j}$. The dark blue bonds corresponding to the highest dimer density are organized into columns. (b) Spin configuration in the first columnar state in the gauge of Fig. 1 (with the same notation for antiferromagnetic bonds). The two types of arrows correspond to the two spin orientations realized in that state. The unit cell is defined by the two vectors $\mathbf{a}$ and $\mathbf{b}$ with $|\mathbf{b}|=\sqrt{3}|\mathbf{a}|$.

based on the expectation that the difference in energy between each pair of states is determined primarily by the difference in the number of domain walls they contain.

In Sec. II B we established that the structure of columnar solutions is described by Eqs. (9), where $\sigma_{i}= \pm 1$ is determined by the ground state of the pure Ising case and the values of the variables $\theta_{i}$ are given by Eqs. (20) and (21). It is convenient to start the construction of the Hamiltonian describing the harmonic fluctuations around these states by performing a rotation of the spins on each site,

$$
\begin{gathered}
S_{i}^{x}=\sigma_{i} \cos \theta_{i} S_{i}^{x \prime}+\sin \theta_{i} S_{i}^{z \prime}, \\
S_{i}^{y}=S_{i}^{y \prime}, \\
S_{i}^{z}=-\sin \theta_{i} S_{i}^{x \prime}+\sigma_{i} \cos \theta_{i} S_{i}^{z \prime}
\end{gathered}
$$

in such a way that the Hamiltonian expressed in terms of the variables $S^{x \prime}$ and $S^{z \prime}$ has a ferromagnetic ground state.

Mapping the new spin operators to Holstein-Primakoff bosons in the harmonic limit, ${ }^{11}$

$$
S_{i}^{z^{\prime}}=S-a_{i}^{\dagger} a_{i}, \quad S_{i}^{x^{\prime}} \approx \sqrt{\frac{S}{2}}\left(a_{i}+a_{i}^{\dagger}\right)
$$


then yields the quadratic Hamiltonian:

$$
\begin{aligned}
H= & E_{\mathrm{col}}+\gamma_{1} \sum_{i \in \mathrm{NF}} a_{i}^{\dagger} a_{i}+\gamma_{2} \sum_{i \in \mathrm{F}} a_{i}^{\dagger} a_{i} \\
& -\frac{J}{2 S} \sum_{\langle i, j\rangle} M_{i j} \sin \theta_{i} \sin \theta_{j}\left[a_{i} a_{j}+a_{i}^{\dagger} a_{j}+\text { H.c. }\right],
\end{aligned}
$$

where

$$
\begin{gathered}
\gamma_{1}=(1 / S)\left[J \cos \theta_{1}\left(2 \cos \theta_{1}+\cos \theta_{2}\right)+\Gamma \sin \theta_{1}\right], \\
\gamma_{2}=(1 / S)\left(J \cos \theta_{1} \cos \theta_{2}+\Gamma \sin \theta_{2}\right),
\end{gathered}
$$

and $E_{\mathrm{col}}$ is the classical energy of a columnar state. Equation (25) can be reduced to a gauge-invariant form (with $M_{i j}$ replaced by $\tau_{i j}$ ) by replacing $a_{i}$ with $\sigma_{i} a_{i}$ and $a_{i}^{\dagger}$ with $\sigma_{i} a_{i}^{\dagger}$ in Eqs. (24). However, we use Eq. (25) in the following because it allows an easy proof that domain walls of the first type do not change the energy of the harmonic fluctuations.

It is evident that for $\theta_{i}$ given by Eq. (20), the expression on the right-hand side of Eq. (25) is exactly the same for all columnar states having the same sets of frustrated and nonfrustrated sites. Since the introduction of domain walls of the first type interchanges only the positions of frustrated and nonfrustrated bonds forming straight columns but does not change the positions of frustrated sites [see Figs. 3(a) and 3(b)], the expression on the right-hand side of Eq. (25) will be exactly the same for all columnar states which can be transformed into one another by introducing some number of domain walls of the first type. This proves that the contribution of the harmonic fluctuations to the energy is the same for all members of the family of columnar states having only domain walls of the first type.

After partitioning the honeycomb lattice into four sublattices in accordance with the structure of the unit cell shown in Fig. 4(b) and performing on each sublattice a Fourier transformation, the quadratic bosonic Hamiltonian of the first columnar state is reduced to the form

$$
H=E_{\mathrm{col}}+\sum_{\mathbf{q}}\left[\mathbf{a}_{\mathbf{q}}^{\dagger} \hat{H}(\mathbf{q}) \mathbf{a}_{\mathbf{q}}-\left(\gamma_{1}+\gamma_{2}\right)\right] .
$$

In this expression, $\mathbf{a}_{\mathbf{q}}$ is an eight-component vector $\left(a_{-\mathbf{q}, 1}, a_{-\mathbf{q}, 2}, a_{-\mathbf{q}, 3}, a_{-\mathbf{q}, 4}, a_{\mathbf{q}, 1}^{\dagger}, a_{\mathbf{q}, 2}^{\dagger}, a_{\mathbf{q}, 3}^{\dagger}, a_{\mathbf{q}, 4}^{\dagger}\right)$, where $a_{\mathbf{q}, n}$ is the bosonic operator with the wave vector $\mathbf{q}$ acting on the $n$th sublattice, and $\hat{H}(\mathbf{q})$ is an $8 \times 8$ Hermitian matrix given by

$$
\hat{H}(\mathbf{q})=\frac{1}{2}\left(\begin{array}{cccccccc}
\gamma_{2} & \mu & 0 & \delta & 0 & \mu & 0 & \delta \\
\mu^{\star} & \gamma_{2} & \tau & 0 & \mu^{\star} & 0 & \tau & 0 \\
0 & \tau & \gamma_{1} & \eta^{\star} & 0 & \tau & 0 & \eta^{\star} \\
\delta^{\star} & 0 & \eta & \gamma_{1} & \delta^{\star} & 0 & \eta & 0 \\
0 & \mu & 0 & \delta & \gamma_{2} & \mu & 0 & \delta \\
\mu^{\star} & 0 & \tau & 0 & \mu^{\star} & \gamma_{2} & \tau & 0 \\
0 & \tau & 0 & \eta^{\star} & 0 & \tau & \gamma_{1} & \eta^{\star} \\
\delta^{\star} & 0 & \eta & 0 & \delta^{\star} & 0 & \eta & \gamma_{1}
\end{array}\right)
$$

where

$$
\begin{gathered}
\mu \equiv \mu(\mathbf{q})=-\frac{J \sin ^{2} \theta_{2}}{2 S}\left(-1+e^{i \mathbf{q a}}\right), \\
\eta \equiv \eta(\mathbf{q})=-\frac{J \sin ^{2} \theta_{1}}{2 S}\left(1+e^{i \mathbf{q a}}\right), \\
\delta \equiv \delta(\mathbf{q})=\tau e^{i \mathbf{q} \mathbf{b}}, \quad \tau=-\frac{J \sin \theta_{1} \sin \theta_{2}}{2 S} .
\end{gathered}
$$

The vectors $\mathbf{a}$ and $\mathbf{b}$ are shown in Fig. 4(b).

As discussed, the harmonic Hamiltonian is the same for the whole family of columnar states constructed by introducing an arbitrary number of domain walls of the first type. This family includes, for instance, the second columnar state. In the harmonic approximation, all these states have the same quantum corrections to the energy; therefore, to order $1 / S$ the degeneracy is not lifted. Note, however, that the absence of degeneracy lifting for this family of states at the harmonic level is not related to a symmetry of the original Hamiltonian. So we expect this degeneracy to be removed if one goes beyond the harmonic approximation, and higher-order terms are expected to select either the first or the second columnar state depending on whether the energy of a domain wall of the first type is positive or negative. However the effect of anharmonicities has not been investigated in this work. Note that a similar effect, namely, the incapacity of harmonic fluctuations to fully lift a well-developed accidental degeneracy of the ground states, has already been reported for various other models (in particular, with kagomé, ${ }^{12-14}$ honeycomb,${ }^{15}$ dice, ${ }^{16}$ and pyrochlore $^{17}$ lattices).

In contrast, the third columnar state is described by a different harmonic Hamiltonian that is not given here explicitly because the number of sites per unit cell, hence the linear dimension of the matrix $\hat{H}(\mathbf{q})$, is twice as large, so that the matrix $\hat{H}(\mathbf{q})$ is $16 \times 16$. The energy of zero point fluctuations in this state turns out to be higher than in the first columnar state (see Fig. 5). This suggests that domain walls of the second type have a positive energy.

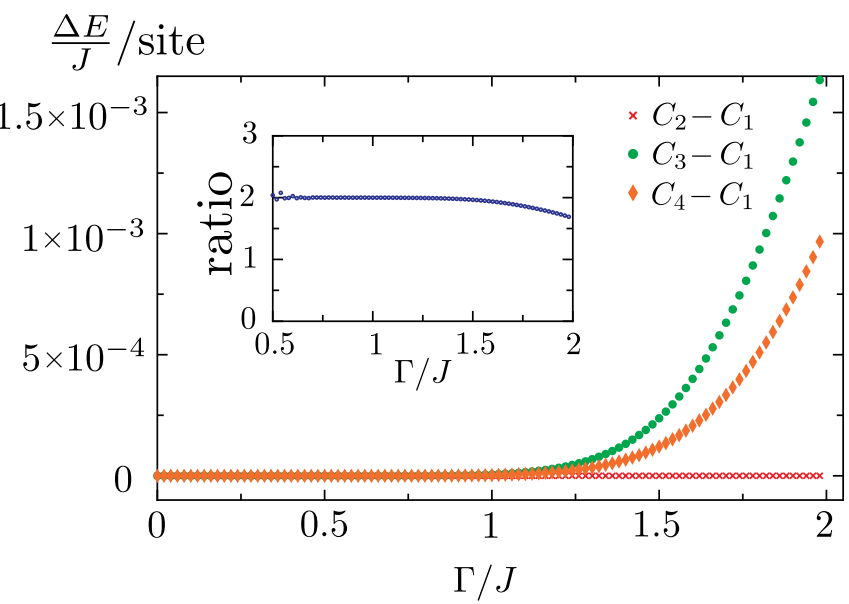

FIG. 5. (Color online) Energies (per site) of the second (red crosses), third (green circles), and fourth (orange diamonds) columnar states calculated in the harmonic approximation, counted with respect to the energy of the first columnar state and expressed in units of $J$. The inset is a plot of the ratio of the energy of the third columnar state over that of the fourth columnar state. 


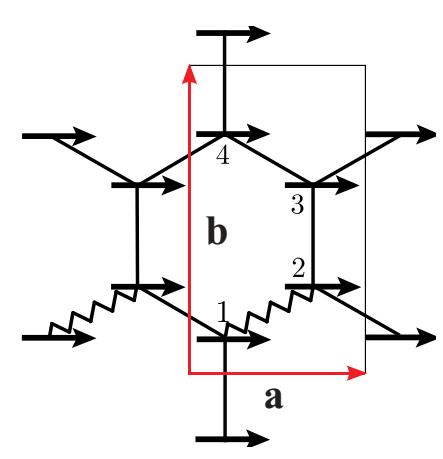

FIG. 6. (Color online) In the polarized state all spins are aligned along the magnetic field. The unit cell of this state is the same as that of the first columnar state: It is defined by vectors $\mathbf{a}$ and $\mathbf{b}$.

To support this statement, we have applied the same reasoning as used in Ref. 18 for the investigation of the frustrated $X Y$ model on a triangular lattice and have considered the fourth columnar state [Fig. 3(d)], which differs from the third one in that the density of domain walls of the second type is exactly half as large. Figure 5 compares the numerically calculated differences between the value of the quantum corrections to the energies of the second, third, and fourth columnar states and its value for the first columnar state. In particular, the inset in Fig. 5 presents the ratio of these quantities for the third and fourth states. This ratio is very close to 2, supporting the suggestion that the fluctuation-induced corrections to the energy are essentially proportional to the density of domain walls of the second type.

When $\Gamma / J$ increases, the classical states remain locally stable until soft modes appear in the spin-wave dispersion. For all columnar states without domain walls of the second type, this takes place at $\Gamma / J \approx 2.004$, and for the third columnar state, it takes place at $\Gamma / J \approx 2.373$. To summarize, harmonic fluctuations partially lift the degeneracy of the classical ground-state manifold in favor of the columnar states having only domain walls of the first type.

\section{PLAQUETTE PHASE}

\section{A. Soft modes and ground-state periodicity}

In the limit $J=0$ the Hamiltonian consists simply of a coupling to the transverse magnetic field $\Gamma$, and the classical ground state is completely polarized, with all spins aligned along the magnetic field in the $x$ direction. The same state minimizes the classical energy for sufficiently large ratio $\Gamma / J$. With the choice of gauge of Fig. 1, the unit cell of this state contains four sites (see Fig. 6).

The analysis of Refs. 7-9 indicates that the polarized phase becomes unstable at $\Gamma=\Gamma_{c}=\sqrt{6} J$. At this value of the field, soft modes appear in the dispersion relation at momenta $\left(q_{x}, q_{z}\right)= \pm\left(\frac{\pi}{6|a|}, \frac{\pi}{2|b|}\right)$ and $\left(q_{x}, q_{z}\right)= \pm\left(\frac{5 \pi}{6|a|}, \frac{\pi}{2|b|}\right)$, triggering a second-order transition to a new phase whose periodicity can be determined from the $\mathbf{q}$ points corresponding to the soft modes.

Since any linear combination of these four modes is invariant under translations by vectors $(3 \mathbf{a}-\mathbf{b})$ and $4 \mathbf{b}$, a state associated with them should have the periodicity in real space imposed by these two vectors that define a unit cell containing

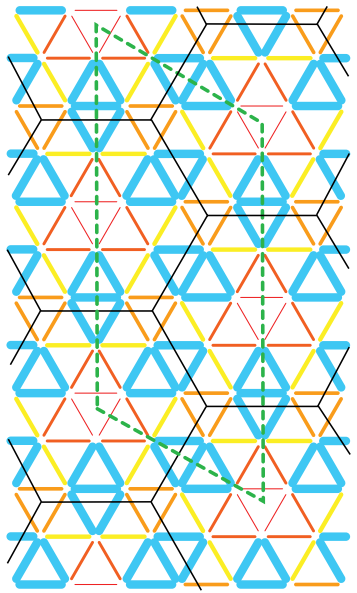

(a) Plaquette phase: dimer representation

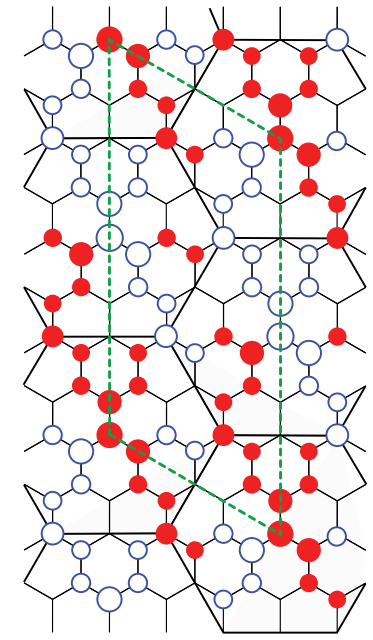

(b) Plaquette phase: unit cell in the spin representation

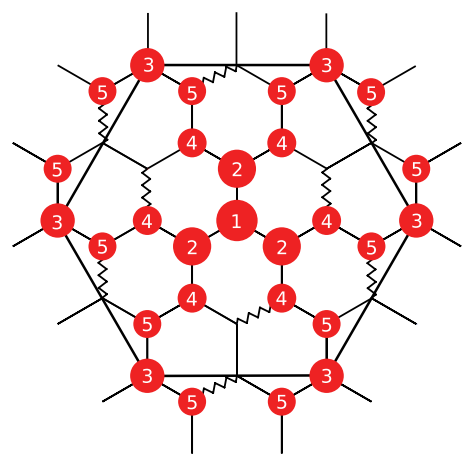

(c) Plaquette phase: the smallest unit cell in the spin representation

FIG. 7. (Color online) (a) The dimer density $d_{i j}$ in the plaquette phase at $\Gamma / J=2$. The thick blue bonds corresponding to the highest density $\left(d_{i j}=\frac{1}{2}\right)$ are organized into four-site rhombic plaquettes. On all other bonds the dimer density satisfies $0<d_{i j}<\frac{1}{2}$, with the thickness of the bonds being proportional to $d_{i j}$. (b) The spin configuration in the same state in the gauge of Fig. 1. The radii of the circles are proportional to $\left|S_{i}^{z}\right|$, while positive and negative values of $S_{i}^{z}$ are represented as full and empty circles. The green dashed parallelogram shows the 48 -site unit cell $(3 \mathbf{a}-\mathbf{b}) \times 4 \mathbf{b}$. It can be split into two halves that differ from each other by the sign of $S^{z}$. (c) The same spin configuration in the gauge that leads to a 24-site unit cell (large hexagon). As before, antiferromagnetic bonds fixing the gauge are depicted as zigzag bonds. The sites at which the classical spins have the same values of $S_{i}^{z}$ are labeled with the same number. Note the existence of six sites with $S_{i}^{z}=0$.

48 sites of the honeycomb lattice [Fig. 7(b)]. Moreover, since any linear combination of the four soft modes under the translation by $2 \mathbf{b}$ just changes sign, this cell should allow a division into two halves that in the spin representation differ from each other only by the reflection of all spins about the $x$ axis but in terms of gauge-invariant variables are identical.

There exists a possibility to make these two halves really identical in terms of spin representation as well just by 
choosing a different gauge, shown in Fig. 7(c). In this gauge a state related to the soft modes listed here is periodic with a 24 -site unit cell defined, for example, by vectors $(3 \mathbf{a}-\mathbf{b})$ and $2 \mathbf{b}$. However, if one uses the simplest gauge of Fig. 1 and imposes periodic boundary conditions along the $x$ and $z$ directions, the periodicity dictated by the wave vectors of the soft modes requires the use a cell of size $12 \mathbf{a} \times 4 \mathbf{b}$ that contains 192 sites of the honeycomb lattice. ${ }^{8}$

\section{B. Numerical minimization of energy}

The minimization of the classical energy using Mathematica minimization routines for the 192-site system with periodic boundary conditions has confirmed that the real periodicity of the classical ground state in the gauge of Fig. 1 is determined by a 48-site unit cell that can be divided into two halves in such a way that the second half differs from the first one by the reflection of all spins about the $x$ axis. Inside the cell one finds a pattern of six different orientations of the spins as well as their reflections about the direction of the field.

The structure of the state minimizing the classical energy is shown in Fig. 7(b). The radii of the circles are proportional to the absolute value of the $z$ component of the spins $\left|S^{z}\right|$, and the different signs of $S^{z}$ are kept track of by plotting full and empty circles. $S^{x}$ is not plotted but is always positive since the spins tend to align with the magnetic field. The size of the elementary cell can be reduced to 24 sites by choosing the gauge depicted in Fig. 7(c) by zigzagged bonds. In this gauge the sign of $S^{z}$ is the same for all spins, and the spin pattern is centered on one of the sites of the honeycomb lattice.

Naturally, it is even more convenient to discuss the structure of an ordered state in terms of gauge-invariant dimer density $d_{i j}$, defined by Eq. (5). In the polarized phase (at $\Gamma / J>\sqrt{6}$ ), $S_{i}^{z}=0$ for all sites $i$, so that the dimer density is uniform and equal to $\frac{1}{2}$ on all bonds. Below the critical magnetic field, $\Gamma_{c}=\sqrt{6} J$, the dimer density on many bonds becomes smaller than $\frac{1}{2}$. For the pattern of $d_{i j}$ the two halves of the 48-site elementary cell are identical because the dimer pattern is conserved when reversing the sign of $S^{z}$ for all spins. Accordingly, the elementary cell corresponds to 24 sites of the honeycomb lattice or to 12 sites of the triangular lattice dual to it. In other terms, the periodicity of the dimer density pattern is the same as in the $\sqrt{12} \times \sqrt{12}$ phase found around $V / t=0$ in the QDM on the triangular lattice. ${ }^{1-4}$

In Fig. 7(a) the elementary cells are represented by the large hexagons. Since the dimer density is the highest on a pattern of four-site plaquettes [with three plaquettes inside elementary cell, see Fig. 7(a)], following the convention adopted in the QDM literature, ${ }^{19}$ we refer to this phase as the plaquette phase. This phase is the analog of the $\sqrt{12} \times \sqrt{12}$ phase found around $V / t=0$ in the QDM. ${ }^{1}$

Note that the dimer density plot obtained below $\Gamma / J=\sqrt{6}$ in our calculation [Fig. 7(a)] differs significantly from the one presented in Ref. 9. The two plots have the same symmetry, $P 31 \mathrm{~m}$, but the pattern of Ref. 9 does not reveal four-site plaquettes. In fact, the difference can be traced back to the fact that the solution of Ref. 9 was obtained by a variational calculation in the subspace of linear combinations of the four soft modes (which minimize the sum of the second- and fourth-order contributions to the classical energy), whereas the present solution was obtained by assuming that the soft modes dictate only its periodicity. The reason why the two solutions do not have the same asymptotic form when $\Gamma / J$ tends to $\sqrt{6}$ from below is detailed in Sec. III C, which is devoted to the analytical investigation of the plaquette state structure in the vicinity of the phase transition.

The degeneracy of the plaquette phase is equal to 48 in terms of the spin representation and to 24 in terms of the dimer representation. Each of the 24 equivalent dimer patterns [one of which is shown in Fig. 7(a)] corresponds to two spin configurations that can be transformed into one another by changing the sign of $S_{i}^{z}$ for all spins.

The local stability of the plaquette phase with respect to quantum fluctuations has been investigated within the gauge of Fig. 7(c) to reduce the Hermitian matrix of the quadratic bosonic Hamiltonian to a $48 \times 48$ matrix. The plaquette phase has been found to be stable in the domain $1.64<\frac{\Gamma}{J}<\sqrt{6}$, with soft modes appearing at $\mathbf{q}=0$ when $\frac{\Gamma}{J} \approx 1.64$.

\section{Analytical study of the critical region below $\boldsymbol{\Gamma}_{c}$}

In this subsection it will be convenient to use a different parametrization of the classical spins of norm $S$ instead of Eqs. (9):

$$
\begin{gathered}
S_{i}^{x}=S \sqrt{1-\rho_{i}^{2}}, \\
S_{i}^{z}=S \rho_{i} .
\end{gathered}
$$

In the asymptotic regime where the transverse field $\Gamma$ dominates over nearest-neighbor interactions, we are in the polarized phase with $S_{i}^{z}=0\left(\rho_{i}=0\right)$. When the transverse field decreases, the components $S_{i}^{z}$ are expected to deviate from zero. To sixth order in $\rho_{i}$, the classical energy of the model is given by

$$
E=-J \sum_{\langle i, j\rangle} M_{i, j} \rho_{i} \rho_{j}-\Gamma \sum_{i}\left(1-\frac{\rho_{i}^{2}}{2}-\frac{\rho_{i}^{4}}{8}-\frac{\rho_{i}^{6}}{16}-\cdots\right)
$$

Let us denote by $\rho_{\mathbf{R}_{i}, n}=\sum_{\mathbf{q}} \rho_{\mathbf{q}, n} e^{i \mathbf{R}_{i} \mathbf{q}}$, with $n=1, \ldots, 4$, the values of $\rho_{i}$ on the four sublattices (see Fig. 6). Since $\rho_{\mathbf{R}_{i}, n}$ is real, $\rho_{\mathbf{q}, n}=\rho_{-\mathbf{q}, n}^{*}$. The energy per site $\mathcal{E}$ is then given by

$$
\begin{aligned}
\mathcal{E}= & \mathcal{E}_{J}=0 \\
& +\frac{J}{8} \sum_{n, n^{\prime}, \mathbf{q}} \rho_{-\mathbf{q}, n}\left[\hat{M}(\mathbf{q})-\frac{\Gamma}{J} \hat{\mathbb{1}}\right]_{n, \mathbf{q}_{1}^{\prime}} \sum_{\mathbf{q}, \mathbf{q}_{2}, \mathbf{q}_{3}, \mathbf{q}_{4}}\left(\prod_{i=1}^{4} \rho_{\mathbf{q}_{i}, n}\right) \delta_{\mathbf{q}_{1}+\mathbf{q}_{2}+\mathbf{q}_{3}+\mathbf{q}_{4}, \mathbf{G}} \\
& +\frac{\Gamma}{64} \sum_{n, \mathbf{q}_{1}, \mathbf{q}_{2}, \mathbf{q}_{3}, \mathbf{q}_{4}, \mathbf{q}_{5}, \mathbf{q}_{6}}\left(\prod_{i=1}^{6} \rho_{\mathbf{q}_{i}, n}\right) \delta_{\mathbf{q}_{1}+\mathbf{q}_{2}+\mathbf{q}_{3}+\mathbf{q}_{4}+\mathbf{q}_{5}+\mathbf{q}_{6}, \mathbf{G}} \\
& +\cdots,
\end{aligned}
$$


where $\mathbf{G}$ is a vector belonging to the reciprocal lattice of the lattice defined by the vectors $\mathbf{a}$ and $\mathbf{b}$ and

$\hat{M}(\mathbf{q})=\left(\begin{array}{cccc}0 & -1+e^{-i q_{x}|a|} & 0 & e^{-i q_{z}|b|} \\ -1+e^{i q_{x}|a|} & 0 & 1 & 0 \\ 0 & 1 & 0 & 1+e^{i q_{x}|a|} \\ e^{i q_{z}|b|} & 0 & 1+e^{-i q_{x}|a|} & 0\end{array}\right)$

is the Fourier transform of the interaction matrix. The analysis of the second-order terms in (33) shows ${ }^{7,8}$ that the paramagnetic solution $\rho_{i}=0$ becomes unstable at $\Gamma / J=\sqrt{6}$ at the wave vectors $\mathbf{q}_{A}=\left(\frac{\pi}{6|\mathbf{a}|}, \frac{\pi}{2|\mathbf{b}|}\right), \mathbf{q}_{B}=\left(\frac{5 \pi}{6|\mathbf{a}|}, \frac{\pi}{2|\mathbf{b}|}\right),-\mathbf{q}_{A}$, and $-\mathbf{q}_{B}$, indicating a transition to a phase of periodicity $(3 \mathbf{a}-\mathbf{b}) \times 4 \mathbf{b}$.

The approach of Ref. 9 consists in keeping in the energy functional (33) only the critical modes with $\mathbf{q}= \pm \mathbf{q}_{A}$ and $\mathbf{q}=$ $\pm \mathbf{q}_{B}$, whose amplitudes are described by Fourier coefficients

$$
\boldsymbol{\rho}_{\mathbf{q}_{A}}=\left|\rho_{A}\right| e^{i \phi_{A}} \mathbf{u}_{A}, \quad \boldsymbol{\rho}_{\mathbf{q}_{B}}=\left|\rho_{B}\right| e^{i \phi_{B}} \mathbf{u}_{B},
$$

where

$$
\begin{aligned}
& \mathbf{u}_{A}=\left(1, e^{i \frac{7 \pi}{12}}, F e^{i \frac{7 \pi}{12}}, F e^{-i \frac{3 \pi}{2}}\right) \\
& \mathbf{u}_{B}=\left(F, F e^{i \frac{11 \pi}{12}}, e^{i \frac{11 \pi}{12}}, e^{-i \frac{3 \pi}{2}}\right)
\end{aligned}
$$

are the eigenvectors of $\hat{M}\left(\mathbf{q}_{A}\right)$ and $\hat{M}\left(\mathbf{q}_{B}\right)$ associated with an eigenvalue equal to $\sqrt{6}$ and

$$
F=2 \sin \frac{5 \pi}{12}=\frac{1+\sqrt{3}}{\sqrt{2}} .
$$

In the framework of this approach, $\mathcal{E}_{0}^{(4)}$, the sum of the second- and fourth-order contributions to Eq. (33), is given by

$$
\begin{aligned}
\mathcal{E}_{0}^{(4)}= & -\frac{1}{2}\left(\Gamma_{c}-\Gamma\right)\left(1+F^{2}\right)\left[\left|\rho_{A}\right|^{2}+\left|\rho_{B}\right|^{2}\right] \\
& +\frac{3 \Gamma}{2} F^{2}\left[\left|\rho_{A}\right|^{2}+\left|\rho_{B}\right|^{2}\right]^{2}
\end{aligned}
$$

and depends only on $\left|\rho_{A}\right|^{2}+\left|\rho_{B}\right|^{2}$ as already noticed in Refs. 7 and 8 .

The minimum of $\mathcal{E}_{0}^{(4)}$ is achieved when

$$
\left|\rho_{A}\right|^{2}+\left|\rho_{B}\right|^{2}=\frac{1+F^{2}}{6 F^{2}} \frac{\Gamma_{c}-\Gamma}{\Gamma},
$$

from which it follows that, to leading order, $\left|\rho_{A}\right| \sim\left|\rho_{B}\right| \sim$ $\left(\Gamma_{c}-\Gamma\right)^{\frac{1}{2}}$ and $\mathcal{E}_{0}^{(4)} \sim\left(\Gamma_{c}-\Gamma\right)^{2}$. However, condition $(38)$ leaves both the ratio $\left|\rho_{B}\right| /\left|\rho_{A}\right|$ and the phases $\phi_{A}$ and $\phi_{B}$ completely undefined. The dependence of $\mathcal{E}$ on these quantities appears if one goes beyond the fourth order and considers also the sixth-order term in Eq. (33), ${ }^{8,9}$ which, for the critical modes, reduces to

$$
\begin{aligned}
\mathcal{E}_{0}^{(6)}= & \frac{5 \Gamma}{8}\left(1+F^{6}\right)\left[\left|\rho_{A}\right|^{2}+\left|\rho_{B}\right|^{2}\right]^{3} \\
& +\frac{3 \Gamma}{2} F^{3}\left[\left|\rho_{A}\right|^{5}\left|\rho_{B}\right| \cos \left(5 \phi_{A}-\phi_{B}\right)\right. \\
& \left.+\left|\rho_{B}\right|^{5}\left|\rho_{A}\right| \cos \left(5 \phi_{B}-\phi_{A}\right)\right] .
\end{aligned}
$$

The general structure of Eq. (39) has been derived in Ref. 8 from the symmetries of the problem.

It follows from the estimate for $\left|\rho_{A}\right|$ and $\left|\rho_{B}\right|$ that to leading order, $\mathcal{E}_{0}^{(6)} \sim\left(\Gamma_{c}-\Gamma\right)^{3}$. For all values of the amplitudes $\left|\rho_{A}\right|$ and $\left|\rho_{B}\right|$, the expression on the right-hand side of Eq. (39) is minimal when both cosines are equal to -1 . This selects the phases

$$
\phi_{A}=\frac{\pi}{6}+\frac{\pi}{12} p, \quad \phi_{B}=-\frac{\pi}{6}+\frac{5 \pi}{12} p,
$$

where $p$ is an integer, yielding 24 independent sets $\left(\phi_{A}, \phi_{B}\right)$. The variation of $\mathcal{E}_{0}^{(6)}$ with respect to $\left|\rho_{A}\right|$ and $\left|\rho_{B}\right|$ under constraints (38) and (40) then selects either $\left|\rho_{B}\right| /\left|\rho_{A}\right|=F$ or $\left|\rho_{B}\right| /\left|\rho_{A}\right|=F^{-1}$. All 48 solutions thus generated correspond to the same dimer pattern (shifted and/or rotated) found in Ref. 9, and thus, we recover the 48-fold degeneracy discussed in Ref. 8.

This approach is based on the assumption that all other modes would only contribute to the energy expansion to higher order. We shall now show that, since one has to push the expansion to order 6 when considering only the critical modes, this assumption is not valid because some second- and fourth-order terms involving noncritical modes also make contributions of order $\left(\Gamma_{c}-\Gamma\right)^{3}$ that are essential for determining $\phi_{A}$ and $\phi_{B}$.

The dominant terms coupling the critical modes with $\mathbf{q}= \pm \mathbf{q}_{A}$ and $\mathbf{q}= \pm \mathbf{q}_{B}$ with extra modes are expected to be linear in the amplitudes of these extra modes and of the third order in the amplitudes of critical modes. The conservation of the total momentum then imposes on the wave vectors of these extra modes the following condition:

$$
\mathbf{q}=m_{A} \mathbf{q}_{A}+m_{B} \mathbf{q}_{B},
$$

where $m_{A}$ and $m_{B}$ are integers and $m_{A}+m_{B}$ is odd. In the first Brillouin zone there are only two wave vectors compatible with this condition: $\mathbf{q}_{C}=2 \mathbf{q}_{A}-\mathbf{q}_{B}$ and $-\mathbf{q}_{C}$. Let us denote the Fourier coefficients associated with the modes with $\mathbf{q}=\mathbf{q}_{C}$ by $\bar{\rho}_{n}=\left|\bar{\rho}_{n}\right| e^{i \bar{\phi}_{n}}$, where $n=1, \ldots, 4$ refers to the number of the sublattice. The terms in the energy functional that are linear and harmonic in $\bar{\rho}_{n}$ are

$$
\begin{aligned}
\mathcal{E}_{1}^{(4)}= & -\frac{J}{4} \sum_{n, n^{\prime}} \bar{\rho}_{n}^{*}\left[\hat{M}\left(\mathbf{q}_{C}\right)-\frac{\Gamma}{J} \hat{\mathbb{1}}\right]_{n, n^{\prime}} \bar{\rho}_{n^{\prime}} \\
& +\frac{\Gamma}{8} \sum_{n=1}^{4}\left(R_{n} \bar{\rho}_{n}+\text { c.c. }\right),
\end{aligned}
$$

with

$$
\begin{aligned}
R_{n}= & \rho_{A}^{3}\left(u_{A}\right)_{n}^{3}+3\left(\rho_{A}^{*}\right)^{2}\left(u_{A}^{*}\right)_{n}^{2} \rho_{B}\left(u_{B}\right)_{n} \\
& +3 \rho_{A}\left(u_{A}\right)_{n}\left(\rho_{B}^{*}\right)^{2}\left(u_{B}^{*}\right)_{n}^{2}+\rho_{B}^{3}\left(u_{B}\right)_{n}^{3} .
\end{aligned}
$$

The variation of Eq. (42) with respect to $\bar{\rho}_{n}^{*}$ gives

$$
\bar{\rho}_{n}=\frac{\Gamma}{2 J} \sum_{n^{\prime}}\left[\hat{M}\left(\mathbf{q}_{C}\right)-\frac{\Gamma}{J} \hat{\mathbb{1}}\right]_{n n^{\prime}}^{-1} R_{n^{\prime}}^{*}
$$

Injecting Eq. (44) into Eq. (42), we obtain

$$
\begin{aligned}
\mathcal{E}_{1}^{(4)}= & -\Gamma h(\Gamma / J)\left[\left|\rho_{A}\right|^{2}+\left|\rho_{B}\right|^{2}\right]^{3} \\
& -\Gamma g(\Gamma / J)\left[\left|\rho_{A}\right|^{5}\left|\rho_{B}\right| \cos \left(5 \phi_{A}-\phi_{B}\right)\right. \\
& \left.+\left|\rho_{A}\right|\left|\rho_{B}\right|^{5} \cos \left(5 \phi_{B}-\phi_{A}\right)\right],
\end{aligned}
$$


where we have introduced the notation

$$
\begin{gathered}
h(\gamma)=\frac{\gamma}{8\left(\gamma^{2}-3\right)}\left\{\gamma\left(1+F^{6}\right)+6 \sqrt{2}\left(3 F^{2}-1\right)\right\}, \\
g(\gamma)=\frac{3 \gamma F}{4\left(\gamma^{2}-3\right)}\left\{4 \gamma F^{2}+3 \sqrt{2}\left(2 F^{2}-1\right)\right\} .
\end{gathered}
$$

Eq. (44) proves that $\bar{\rho}_{n}$ scales as

$$
\left|\bar{\rho}_{n}\right| \sim\left|\rho_{A}\right|^{3} \sim\left|\rho_{B}\right|^{3} \sim\left(\Gamma_{c}-\Gamma\right)^{\frac{3}{2}},
$$

leading to $\mathcal{E}_{1}^{(4)} \sim\left(\Gamma_{c}-\Gamma\right)^{3}$. So it is clear that this contribution cannot be neglected since it is of the same order as $\mathcal{E}_{0}^{(6)}$ and that other contributions involving noncritical modes such as, e.g., sixth-order terms will be of higher order. This means that the phases of the critical modes have to be determined by minimizing the sum of $\mathcal{E}_{0}^{(6)}$ and $\mathcal{E}_{1}^{(4)}$. The contribution to this expression depending on the phases reads

$$
\begin{aligned}
& -\Gamma\left[g\left(\frac{\Gamma}{J}\right)-\frac{3}{2} F^{3}\right]\left[\left|\rho_{A}\right|^{5}\left|\rho_{B}\right| \cos \left(5 \phi_{A}-\phi_{B}\right)\right. \\
& \left.+\left|\rho_{A}\right|\left|\rho_{B}\right|^{5} \cos \left(5 \phi_{B}-\phi_{A}\right)\right] .
\end{aligned}
$$

Now $g(\Gamma / J)-(3 / 2) F^{3}$ is positive for $\Gamma / J>\sqrt{3}$. Therefore, since we are interested in the domain just below $\Gamma / J=\sqrt{6}$, the energy is minimal when both cosines are equal to +1 . This selects the phases

$$
\phi_{A}=\frac{\pi}{12} p, \quad \phi_{B}=\frac{5 \pi}{12} p,
$$

where $p$ is an integer. This leads again to 24 independent sets $\left(\phi_{A}, \phi_{B}\right)$. In addition, minimizing $\mathcal{E}_{0}^{(6)}+\mathcal{E}_{1}^{(4)}$ with respect to the amplitudes $\left|\rho_{A}\right|$ and $\left|\rho_{B}\right|$ under constraint (38) selects, as before, either $\left|\rho_{B}\right| /\left|\rho_{A}\right|=F$ or $\left|\rho_{B}\right| /\left|\rho_{A}\right|=F^{-1}$. The 48 resulting solutions correspond to the 24 equivalent dimer patterns that can be obtained from the one shown in Fig. 7(a). The difference between Eqs. (40) and (47) explains the qualitative difference between the structures of the plaquette phase found in this work and the solution of Ref. 9, which does not disappear even when the amplitudes of the $\mathbf{q}= \pm \mathbf{q}_{C}$ modes become negligible as compared to those of the critical modes.

\section{INTERMEDIATE MIXED PHASES}

During the numerical minimization of the classical energy for the 192-site system with periodic boundary conditions, an additional intermediate phase was found to exist between the columnar and the plaquette phases. We refer to this intermediate phase as the mixed phase because in the dimer representation the bonds with larger dimer densities, $d_{i j} \geqslant \frac{1}{2}$, are arranged in an alternating pattern of plaquettes and columns [see Fig. 8(a)]. The mixed and plaquette phases have the same translational symmetries. However, the point-group symmetries of the gauge-invariant dimer patterns in the two phases are different: $P 31 \mathrm{~m}$ for the plaquette phase [see Fig. 7(a)] and $\mathrm{Cmm}$ for the mixed phase [see Fig. 8(a)]. The phase transition between these two phases has to be of the first order since the symmetry groups are not such that one is a subgroup of the other.

As in the case of the plaquette phase, the size of a unit cell of the mixed phase can be reduced from 48 sites for the standard gauge shown in Fig. 1 to 24 sites in the gauge in Fig. 7(c); see Fig. 8(b). In this gauge the spin pattern consists of spins with the same sign of $S_{i}^{z}$ having seven different orientations, one of which is in the direction of the field. In contrast to the spin pattern in the plaquette phase, which is centered on one of the sites of the honeycomb lattice [Fig. 7(c)], in the mixed phase this pattern is centered on one of the bonds of the lattice [Fig. 8(b)], which explains the difference in symmetry between the two states.

The degeneracy of the mixed phase is equal to 36 in terms of the dimer representation and to 72 in terms of the spin representation. Each of the 36 equivalent dimer patterns corresponds to two spin configurations that can be transformed into one another by changing the sign of $S_{i}^{z}$ for all spins. The stability of the mixed state with respect to small fluctuations has been investigated with LSWT in the gauge producing a 24-site unit cell, and this phase has been found to be stable in the range $1.394 \lesssim \Gamma / J \lesssim 1.774$.

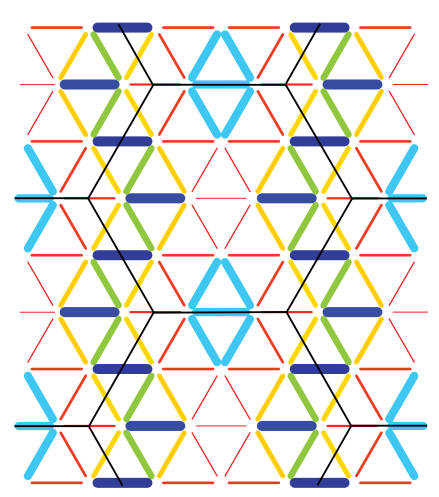

(a) Mixed phase: dimer representation

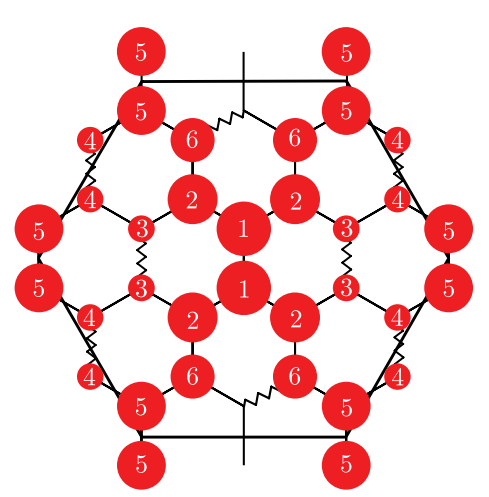

(b) Mixed phase: spin representation

FIG. 8. (Color online) (a) The dimer density $d_{i j}$ in the mixed phase at $\Gamma / J=1.72$. The thickness of the bonds is proportional to $d_{i j}$. The dimer densities are also emphasized by the colors of the bonds ranging from red (light gray) (the lowest densities) to dark blue (dark gray) (the highest densities, $d_{i j}>\frac{1}{2}$ ). The bonds with $d_{i j} \geqslant \frac{1}{2}$ are organized in an alternating pattern of plaquettes and columns. (b) The spin configuration in the same state in the gauge that leads to a 24-site unit cell (large hexagon). Note the existence of two sites at which $S_{i}^{z}=0$. 

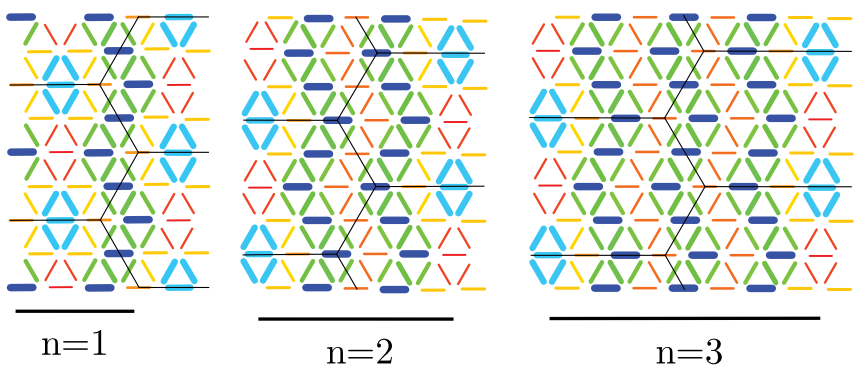

FIG. 9. (Color online) Dimer patterns in the mixed states with $n \leqslant 3$, where $n$ denotes the number of columns separating the plaquette patterns. The notation is the same as in Figs. 7(a) and 8(a). The bonds with $d_{i j} \geqslant \frac{1}{2}$ are organized in an alternating pattern of plaquettes and columns. Thin lines show the boundaries between unit cells.

The existence of the mixed state whose structure is shown in Fig. 8 suggests that there can also exist states in which the straight rows of plaquettes are still equidistant but are separated not by single columns but by a larger number of columns, denoted by $n$ (see Fig. 9). From here on, we number such mixed states by the index $n$ and call the simplest mixed state discussed in the beginning of this section the first mixed state.

It is not hard to understand that the unit cell of the second mixed state (in the optimal gauge in which the sign of $S^{z}$ is the same for all spins) has exactly the same symmetry as the unit cell of the first mixed state and can be obtained from it by adding eight more sites on each side. The successive repetition of this procedure allows one to construct the unit cell for any integer $n$ and to find that it contains $8(2 n+1)$ sites. However, due to the symmetry of the unit cell, the number of nonequivalent sites only increases by four when $n$ increases by one, which leads to $4 n+3$ nonequivalent sites.

For $n \leqslant 7$, we have performed a numerical minimization of the energy for the unit cells corresponding to such structures, and we have found that, when $\Gamma / J$ decreases, the energy of the second mixed state first becomes lower than that of the first mixed state, after which the energy of the third mixed state becomes lower than that of the second mixed state, and so on. Table I summarizes the values of $\Gamma / J$ at which the transition between the $n$th and $(n+1)$ th mixed states takes

TABLE I. The second column shows $\Gamma_{c}^{n, n+1}$, the critical fields at which the transitions between the $n$th and $(n+1)$ th mixed states would take place if there were no other more complex states (which is not always true). The third column shows the width of the field intervals in which the $n$th mixed state has the lowest energy.

\begin{tabular}{lcc}
\hline \hline$n$ & $\Gamma_{c}^{n, n+1} / J$ & $\Delta \Gamma^{n} / J$ \\
\hline 1 & 1.69372479498 & $4.3 \times 10^{-2}$ \\
2 & 1.67655449242 & $1.7 \times 10^{-2}$ \\
3 & 1.67613666486 & $4.2 \times 10^{-4}$ \\
4 & 1.67612802659 & $8.6 \times 10^{-6}$ \\
5 & 1.67612786551 & $1.6 \times 10^{-7}$ \\
6 & 1.67612786267 & $2.8 \times 10^{-9}$ \\
7 & $\cdots$ & $\cdots$ \\
\hline \hline
\end{tabular}

place and reports the width of the region in which the $n$th mixed state has the lowest energy. It can be seen that for $n>1$ this width is scaled down by a factor of the order of 50 each time $n$ increases by 1 . This means that $\Gamma_{c}^{n, n+1}$ approaches a finite limit exponentially fast. The extrapolation shows that the accumulation point of $\Gamma_{c}^{n, n+1}$ at $n \rightarrow \infty$ is $\Gamma_{c}^{\infty} / J=1.67612786261$. Below this field columnar states have the lowest classical energy.

Note that it was impossible to discover any of the mixed states with $n>1$ during the minimization of the energy for the 192-site cell (with periodic boundary conditions and the standard gauge of Fig. 1) which was instrumental in discovering the $n=1$ mixed state. The reason is very simple: The periodicity of all the states with $n>1$ is incompatible with the periodic boundary conditions implemented in this 192-site cell.

The existence of such a sequence of phase transitions suggests that the main contribution to the energy of the $n$th mixed phase (counted off from the energy of a columnar state) is proportional to the density of linear defects (vertical rows of plaquettes) whose energy can be considered as linearly dependent on $\Gamma$, whereas the main correction to this energy comes from the repulsion of nearest defects, which decreases exponentially fast with the distance between them. This was checked at $\Gamma=\Gamma_{c}^{\infty}$, where the proper energy of a linear defect changes sign, and, indeed, we have found that the energies of different states are compatible with an interaction of linear defects that is exponential in the distance between them. This makes us confident that the narrow region above $\Gamma_{c}^{\infty}$ has to contain an infinite sequence of mixed phases with all integer indices $n$.

It is well known that in a system consisting of a sequence of linear defects there can also exist phases with more complex structures, in which the linear defects are not equidistant. In terms of our problem such phases would correspond to a regular alternation of, for example, $n$ and $n+1$ columns or of $n, n$ and $n+1$ columns, etc., leading to what is known as a devil's staircase. ${ }^{20}$ Usually, such phases appear in a phase diagram if the interaction of more distant defects is also repulsive, whereas when the interaction between nextto-nearest defects is attractive, one gets a direct transition from the $n$th to the $(n+1)$ th phase without the presence of an intermediate $(n, n+1)$ phase.

We have verified numerically that in our system the energy of the $(1,2)$ mixed state is never lower than either the energy of the first state or that of the second mixed state, which means that it cannot be present in the phase diagram. Quite surprisingly, the situation with the $(2,3)$ phase is different, and in a narrow interval around $\Gamma_{c}^{2,3}$ (from $\Gamma_{c}^{2,3}-1.3 \times 10^{-9}$ to $\Gamma_{c}^{2,3}+1.9 \times 10^{-9}$ ), its energy is lower than the energies of the second and third mixed states. One can estimate that even if some other complex phases do exist, the field range where any of them minimizes the energy will be at least a couple of orders of magnitude smaller than the already extremely narrow interval of the existence of the $(2,3)$ state, so we decided not to pursue the investigation of this point any further since it cannot be of much relevance.

A more important question is whether the plaquette and the first mixed states may be separated by a region where mixed states of a different type appear, in which the density 
of columns is lower than in the first mixed state, so that the neighboring columns are separated by domains of the plaquette state. Such a scenario seems to us to be impossible, however, for the following reasons.

The comparison of Fig. 7(c) with Fig. 8(b) suggests that the structure of the first mixed state is very close to what one would obtain by constructing the superposition of two plaquette states centered on neighboring sites of the lattice (and letting this superposition relax). Therefore, one can interpret these two states as different manifestations of a unique state that can move around in a complex periodic potential with minima both at the positions corresponding to lattice sites and at the positions corresponding to the middles of lattice bonds. For $\Gamma>\Gamma_{c}^{0,1}=1.73690830184 J$, the minima located at lattice sites are the lowest, whereas for $\Gamma<\Gamma_{c}^{0,1}$, the minima located at the middle of lattice bonds are the lowest. Exactly at $\Gamma=\Gamma_{c}^{0,1}$ all these minima have equal depths. This idea can be confirmed by constructing a family of states that continuously interpolates between the plaquette and the first mixed state, which allows a numerical analysis of the effective potential discussed here. This analysis reveals that at $\Gamma=\Gamma_{c}^{0,1}$ the barrier separating unequivalent (but equal) minima is very low $\left(\sim 1.07 \times 10^{-5} \mathrm{~J}\right.$ per site $)$. Nonetheless, any attempt to construct a state that looks like the plaquette state in some places and like the first mixed state in other places would force the system to overcome this barrier in the intermediate regions. This would increase its energy in comparison with that of the plaquette or of the first mixed state.

The numerical evidence in favor of this conclusion comes from observing that the state that would differ from the first mixed state by having half its density of columns has a periodicity that is compatible with the 192-site cell used in our numerical energy minimization. Therefore, if at $\Gamma=\Gamma_{c}^{0,1}$ the energy of this state was lower than that of the plaquette and of the first mixed states, this state would be accessible during this minimization procedure. To be on the safe side, we have also performed a minimization of the energy for the cell whose periodicity, in addition to the formation of the plaquette and of the first mixed states, allows for the appearance of the states that differ from the first mixed state by keeping only one column out of three (or two out of three), but this has not allowed us to find any state with energy lower than that of the plaquette or of the first mixed state. This gives additional evidence in favor of our conclusion that the phase transition between the plaquette and the first mixed states should be a direct one without any intermediate phases with a more complex structure.

\section{PHASE DiAgRAM}

\section{A. Classical phase diagram}

The classical phase diagram consists of four regions: (i) the columnar phase, which is highly degenerate since all columnar states have the same energy and which extends up to $\Gamma / J \approx$ 1.676 , (ii) the region of mixed states with columnar patterns separated by straight rows of plaquettes in the interval $1.676 \lesssim$ $\Gamma / J \lesssim 1.737$, (iii) the plaquette phase, with a 24 -site unit cell, in the range $1.737 \lesssim \Gamma / J \leqslant \sqrt{6} \approx 2.45$, and (iv) the fully polarized phase with all spins pointing in the direction of the field for $\Gamma / J>\sqrt{6}$. The transition from the fully polarized phase to the plaquette phase is a second-order one, with all other transitions being of the first order. These results are summarized in Fig. 10.

\section{B. Quantum fluctuations}

Quantum fluctuations can a priori modify this phase diagram in two main ways. First of all, if the degeneracy of the classical ground states is accidental (that is, not related to symmetry), they can select some of these states. This is, indeed, the case in the columnar phase, where the columnar states with domain walls of only the first type are selected already at the level of harmonic fluctuations.

Second, quantum fluctuations can shift the phase boundaries. When one takes into account only the harmonic fluctuations, this applies only to first-order transitions. Indeed, at a first-order transition, the classical energy is the same for the two competing configurations, but the spectra of harmonic fluctuations are different, and one phase will, in general, be stabilized over the other by zero-point fluctuations. A convenient way to keep track of the stability of the various phases with respect to quantum fluctuations is to draw a phase
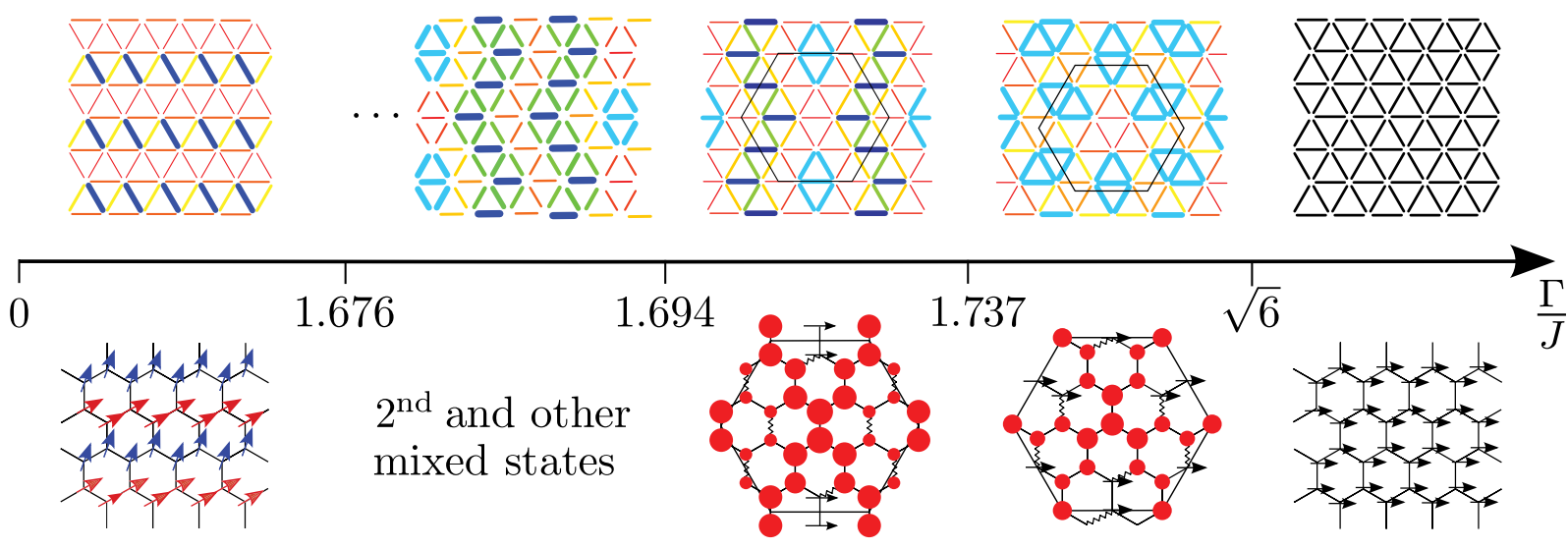

FIG. 10. (Color online) Classical phase diagram (top) in the dimer language and (bottom) in the spin language. In the dimer representation the thickness of the bonds is proportional to the dimer density. Thick blue bonds correspond to the highest dimer density. In the spin representation the radii of the circles are proportional to $S_{i}^{z}$, and arrows indicate the orientation of the classical spins. 


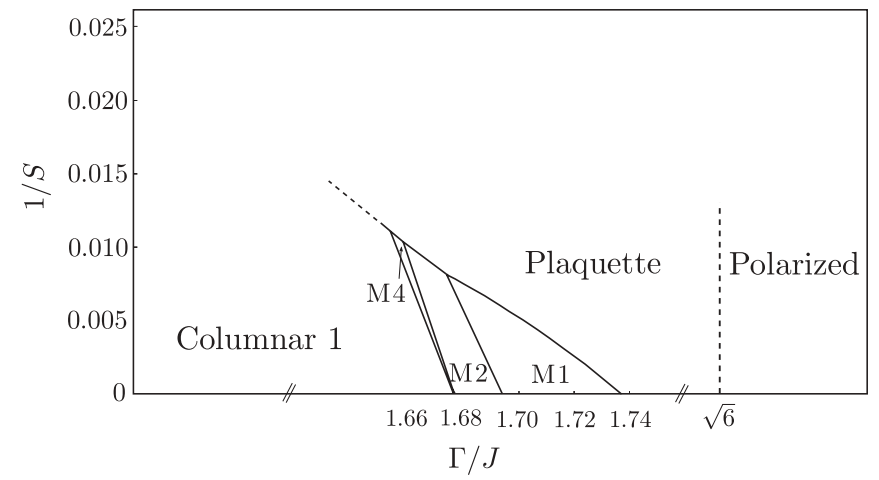

FIG. 11. Semiclassical phase diagram zoomed for values of the field close to the accumulation point of mixed states. $M n$ with $n \in$ $\{1,2,4\}$ denote first, second, and fourth mixed states.

diagram in the $(\Gamma / J, 1 / S)$ plane (see Fig. 11), showing which phase has the lowest total energy.

The resulting phase diagram can be quite involved when there are many phases in competition, and this is clearly the case here since, for $1 / S=0$, there exists an infinite sequence of mixed phases. However, it turns out that for $1 / S$ greater than $10^{-3}$, only three of them survive, as is shown in Fig. 11. All other mixed phases exist only for $1 / S \lesssim 10^{-4}$ in a very narrow range of transverse magnetic field of width $\lesssim 10^{-4} \mathrm{~J}$. They are thus invisible on the scale of Fig. 11, which has been adjusted to properly describe the competition between the two main phases (plaquette and columnar). On that scale, the phase diagram consists of six phases: the polarized phase; the plaquette phase; the first, second, and fourth mixed states; and the columnar phase. The general trend is that the plaquette phase is stabilized by quantum fluctuations over the mixed phases as well as over the columnar phase.

Note that the transition between the plaquette and the columnar phases cannot be followed below $\Gamma / J=1.64$ at this level of approximation because the plaquette phase is no longer locally stable with respect to harmonic fluctuations. The continuation of this boundary by a dashed line in Fig. 11 is just a guide to the eye. To follow this line further would require to go beyond the harmonic approximation. The transition between the plaquette and polarized phases being of the second order, the boundary has to start vertically since, at the transition, both states have the same quantum corrections in the harmonic approximation. This is indicated by a vertical dashed line in Fig. 11. To find the curvature of this line would require going beyond the harmonic approximation.

In view of the very strong modification of these phase boundaries with decreasing $S$, it is legitimate to question the fate of the columnar and mixed phases for $S=1 / 2$, for which the model can be mapped onto the QDM in the limit $\Gamma / J \rightarrow 0$. The results presented here suggest that the mixed phases have absolutely no chance of extending to $S=\frac{1}{2}$.

Regarding the competition between the columnar and the plaquette phases, we can get an estimate of the critical value of the spin at which the boundary between them crosses the axis $\Gamma=0$ by looking at the linear $1 / S$ corrections, starting from the point where the two phases have the same classical energy, a point that does not appear on the phase diagram of Fig. 11 since it lies inside the first mixed phase. This leads to the conclusion that the columnar phase disappears above $1 / S \approx 0.67$, i.e., below $S \approx 1.49$. Note that this should probably be considered as a lower bound in terms of $S$ since the boundary is slightly concave. So, for $S=1 / 2$, the semiclassical calculation at the harmonic level predicts only two phases: a plaquette phase up to $\Gamma / J=\sqrt{6}$ and a polarized phase above that. The fact that we find the point $\Gamma / J=0$ to be in the region of stability of the plaquette phase is in good agreement with the QDM, which has been found by QMC to be in the $\sqrt{12} \times \sqrt{12}$ phase at $V / t=0 .{ }^{1,2}$

\section{CONCLUSIONS}

In conclusion, we have investigated the classical phase diagram of the FFTFIM on the honeycomb lattice and how it is modified by the semiclassical corrections induced by harmonic fluctuations. Compared to what has been already known about the model, namely, that the paramagnetic phase is unstable at $\Gamma / J=\sqrt{6}$ toward a crystalline phase with a large unit cell, the classical phase diagram turns out to be surprisingly rich, with a multitude of additional phases: a columnar phase at a small transverse field and an infinite cascade of phases of mixed columnar and plaquette character. The phase toward which the paramagnetic phase is unstable at $\Gamma / J=\sqrt{6}$ has been found to have the same symmetry and periodicity as the state proposed in Ref. 9, but with a different structure. Both are characterized by a 24 -site unit cell in the spin language and by a 12-site cell on the dual lattice in the dimer language, but the state we have found has a plaquette structure. At the classical level, the columnar phase is fully degenerate, with all columnar states having rigorously the same classical energy.

Quantum fluctuations have been found to modify this phase diagram in two important respects. First of all, harmonic fluctuations have been shown to partially lift the degeneracy of the columnar phase in favor of the columnar states with only one type of domain walls. Since the remaining degeneracy is not related to a symmetry of the model, anharmonic corrections are expected to lift this degeneracy further. Second, they strongly modify the phase boundaries, and for the ultraquantum limit, $S=1 / 2$, they predict that the plaquette phase survives down to $\Gamma \rightarrow 0$.

Going back to the original motivation for this investigation, namely, the properties of the QDM on the triangular lattice, a number of comments should be made about these results. First of all, our semiclassical approximation predicts that the phase that is the analog of the $\sqrt{12} \times \sqrt{12}$ phase of the QDM has a four-site plaquette structure. This reopens the issue of the nature of the $\sqrt{12} \times \sqrt{12}$ phase of the QDM. According to the results of GFQMC simulations, ${ }^{3}$ possible structures are constrained by a quasiextinction of the dimer density correlation function at the corner of the Brillouin zone. This has been shown to be consistent with a uniform distribution of dimer density inside the interior part of the 12-site hexagonal unit cell, a conclusion somehow supported by the conclusions of Ref. 9 regarding the nature of the phase close to the paramagnetic phase. Now that we know that this phase is, in fact, a plaquette phase, it would be interesting to revisit the GFQMC 
results to see to what extent a plaquette phase of this type might be consistent with the quasiextinction at the zone corner.

It is also inspiring that a columnar phase appears in the classical solution of the FFTFIM since a similar phase is present in the QDM for attractive interactions between dimers. We did not manage to find a convincing connection between large $S$ in the FFTFIM and negative $V$ in the QDM, but since we found intermediate phases between the columnar phase and the plaquette phase in the FFTFIM, it is tempting to speculate that such phases may also exist in the QDM.

\section{ACKNOWLEDGMENTS}

The authors acknowledge useful discussions with G. Misguich and financial support from the Swiss National Fund and MaNEP. S.E.K. acknowledges also the support from the RFBR Grant No. 09-02-01192-a.

\section{APPENDIX: COMPARISON OF COLUMNAR AND STAGGERED STATES}

Columnar states are the states that maximize $N_{d}$, the number of pairs of frustrated bonds situated at the smallest possible distance from each other [as shown in Fig. 2(d)]. In this Appendix we want to compare the classical energy of these states with the energy of the states in which $N_{d}$ is minimal, that is, equal to zero. In terms of dimer models such states are usually called staggered or nonflippable states ${ }^{1}$ because they do not contain flippable pairs of dimers.

Since in a staggered state all frustrated sites have identical environments (with exactly one frustrated neighbor) and all nonfrustrated sites also have identical environments (with exactly two frustrated neighbors), such a state can be described by the same two variables $\theta_{1}$ and $\theta_{2}$ introduced in Sec. II B for the description of a columnar state. In terms of $\theta_{1}$ and $\theta_{2}$ the energy of a staggered state can be written as

$$
\begin{aligned}
E_{\text {st }}= & -\frac{N}{2}\left[\frac{J}{2}\left(\cos ^{2} \theta_{1}+4 \cos \theta_{1} \cos \theta_{2}-\cos ^{2} \theta_{2}\right)\right. \\
& \left.+\Gamma\left(\sin \theta_{1}+\sin \theta_{2}\right)\right] .
\end{aligned}
$$

Even without minimizing $E_{\text {st }}$ with respect to $\theta_{1}$ and $\theta_{2}$, one can note that for any $\theta_{1}$ and $\theta_{2}$

$$
E_{\mathrm{st}}\left(\theta_{1}, \theta_{2}\right)-E_{\mathrm{col}}\left(\theta_{1}, \theta_{2}\right)=(J N / 4)\left(\cos \theta_{1}-\cos \theta_{2}\right)^{2} \geqslant 0,
$$

and therefore, the energy of a staggered state [the minimum of $\left.E_{\mathrm{st}}\left(\theta_{1}, \theta_{2}\right)\right]$ has to be higher than the energy of a columnar state [the minimum of $E_{\mathrm{col}}\left(\theta_{1}, \theta_{2}\right)$ achieved when $\cos \theta_{1} \neq \cos \theta_{2}$ ]. This proves that the maximization of $N_{d}$ is always a better strategy than its minimization, even when the ratio $\Gamma / J$ is not small.
${ }^{1}$ R. Moessner and S. L. Sondhi, Phys. Rev. Lett. 86, 1881 (2001).

${ }^{2}$ A. Ralko, M. Ferrero, F. Becca, D. Ivanov, and F. Mila, Phys. Rev. B 71, 224109 (2005).

${ }^{3}$ A. Ralko, M. Ferrero, F. Becca, D. Ivanov, and F. Mila, Phys. Rev. B 74, 134301 (2006).

${ }^{4}$ A. Ralko, M. Ferrero, F. Becca, D. Ivanov, and F. Mila, Phys. Rev. B 76, 140404 (2007).

${ }^{5}$ J. Villain, J. Phys. C 10, 1717 (1977).

${ }^{6}$ B. K. Chakrabarti, A. Dutta, and P. Sen, Quantum Ising Phases and Transitions in Transverse Ising Models (Springer-Verlag, Berlin, 1996).

${ }^{7}$ R. Moessner, S. L. Sondhi, and P. Chandra, Phys. Rev. Lett. 84, 4457 (2000).

${ }^{8}$ R. Moessner and S. L. Sondhi, Phys. Rev. B 63, 224401 (2001).

${ }^{9}$ G. Misguich and F. Mila, Phys. Rev. B 77, 134421 (2008).

${ }^{10}$ R. Moessner, S. L. Sondhi, and E. Fradkin, Phys. Rev. B 65, 024504 (2001).
${ }^{11}$ T. Holstein and H. Primakoff, Phys. Rev. 58, 1098 (1940).

${ }^{12}$ A. B. Harris, C. Kallin, and A. J. Berlinsky, Phys. Rev. B 45, 2899 (1992).

${ }^{13}$ J. T. Chalker, P. C. W. Holdsworth, and E. F. Shender, Phys. Rev. Lett. 68, 855 (1992).

${ }^{14}$ I. Ritchey, P. Chandra, and P. Coleman, Phys. Rev. B 47, 15342 (1993).

${ }^{15}$ S. E. Korshunov and B. Douçot, Phys. Rev. Lett. 93, 097003 (2004).

${ }^{16}$ S. E. Korshunov, Phys. Rev. B 71, 174501 (2005); Phys. Rev. Lett. 94, 087001 (2005).

${ }^{17}$ C. L. Henley, Phys. Rev. Lett. 96, 047201 (2006).

${ }^{18}$ S. E. Korshunov, A. Vallat, and H. Beck, Phys. Rev. B 51, 3071 (1995).

${ }^{19}$ R. Moessner and K. S. Raman, in Highly Frustrated Magnetism, edited by C. Lacroix, P. Mendels, and F. Mila (Springer-Verlag, Heidelberg, Germany, 2010); e-print arXiv:0809.3051 and references therein.

${ }^{20}$ P. Bak, Rep. Prog. Phys. 45, 587 (1982). 